\title{
Performance Driven Multi-Layer General Area Routing for PCB/MCM Designs
}

\author{
Jason Cong and Patrick H. Madden \\ UCLA Computer Science Department \\ Los Angeles, California, 90095
}

\begin{abstract}
In this paper we present a new global router appropriate for Multichip Module (MCM) and dense Printed Circuit Board (PCB) design, which utilizes a hybrid of the classical rip-up and reroute approach, and the more recent iterative deletion[9] method. The global router addresses performance issues by utilizing recent results in high performance interconnect design, while still effectively minimizing global congestion.

With experiments on the maze-routing component of our global router, we show that the choice of routing cost functions can have a significant impact on final solution quality. The results of a number of previously proposed routers may be improved dramatically by adopting the cost functions we suggest here. We also find little evidence of the "net ordering problem" when our cost functions and routing model are applied. The iterative deletion method is shown to improve global solution quality, particularly when high performance interconnect is required. We evaluate the performance of our global router by comparing the congestion of routes produced by our global router to those of a well known MCM router, V4R [14].

Our global router, MINOTAUR, supports arbitrary numbers of routing layers, differing capacities for each layer, preexisting congestion and obstacles, and high performance interconnect structures (including those which require variable width interconnect).
\end{abstract}

\section{Introduction}

Advances in VLSI fabrication technology have resulted in an increasing interest in interconnection and packaging technologies. For many systems, the delay due to circuit interconnections can dominate the total system delay $[1,5]$. Interconnect optimization must be considered to achieve high performance at the system level.

One approach that provides many benefits to packaging level interconnection is the Multichip Module (MCM) technology. This technology increases practical packing densities, and removes a level of interconnection. In MCM designs, a number of bare chips are placed directly onto a substrate, with the substrate itself providing the circuit interconnections. Routing problems for MCM designs have many similarities to routing for dense Printed Circuit Boards (PCBs).

Routing within MCM substrates or PCBs, however, can be an extremely difficult problem with a potentially large number of interconnect layers. A Fujitsu supercomputer design, for example, has over 50 interconnect layers, each with a large routing capacity [10]. The $\mathrm{MCM} / \mathrm{PCB}$ routing problem in general may be considered to be an extremely large general area routing problem, and cannot be decomposed into small channel or switchbox routing problems easily. Additionally, high performance designs must consider signal crosstalk (due to coupling capacitance between long parallel lines), and variable width wiring (to address impedance mismatches and wiring termination requirements).

The problem of MCM/PCB routing has been considered by a number of authors. One set of approaches perform global and detail routing in a single step.

An early MCM router was presented in [10], and performs a combination of $2 \mathrm{D}$ and $3 \mathrm{D}$ routing obtain a solution. Computation times for this method were relatively high.

In [13] the SLICE router was presented; it constructs detailed routes through a mixture of planar routing and twolayer maze routing. While this router was superior to a $3 \mathrm{D}$ maze router, it requires relatively high computation times. The V4R router [14] was subsequently presented, and efficiently routes layer-pairs one at a time using routes with no more than four vias each.

The $M^{2} R[4]$ router addresses performance concerns by routing critical nets on a single layer. The remaining nets are routed using $x-y$ layer pair routing.

In [17], a linear programming approach was presented. Randomized rounding is used to obtain integer solutions. A small number of possible topologies are considered, and routes are restricted to a channel intersection graph.

The MCG [2] router considers a small number of possible routes for each net, and attempts to maximize the routing density on any layer-pair by selecting a set of compatible routes from among the unrouted nets. The results of this router are quite good, requiring fewer routing layers than many of the other MCM routers. Performance concerns are addressed by removing nets which exceed signal crosstalk thresholds, and routing them on other layers.

In [20], the MLR router was presented; it first performs layer assignment of nets, followed by Steiner optimal area routing using a hierarchical $(\alpha, \beta)$ approach (to find lowcost paths between pins).

The SEGRA [3] MCM global router has been described, and also approaches the routing problem by completing pairs of layers in each pass. A simple greedy heuristic is used to select which nets are to be extended or completed during each pass.

While the methods mentioned above consider global and detail routing in a single step, there have also been approaches which consider the problem in two steps.

In [21], the MCM substrate is decomposed into a set of "towers," rectangular regions containing portions of the routing surface through all layers. They first distribute routing density across a three dimensional surface, using hierarchical decomposition, then determine locations of nets on 
the boundaries of the towers, and finally obtain a routing for each tower using a detail router.

We approach the problem in a manner similar to [21], and consider the global routing problem in this paper. In contrast to these previous approachs, our MINOTAUR global router offers the following features.

- We utilize current high-performance variable-width interconnect optimization results, obtaining structures which address delay and signal integrity requirements [6]. We can in fact consider a set of high performance interconnect structures for each signal net, and perform congestion optimization from a global perspective.

- We optimize global congestion by not restricting the layers a route may use; some approaches route a single layer-pair at a time, and cannot obtain routing solutions which span multiple or non-paired layers.

- We combine the freedom and flexibility of maze routing solutions, with the global optimization abilities of the iterative deletion method.

While we consider MCM and PCB routing in this paper, the basic techniques used in our router can be easily applied to multilayer VLSI IC routing problems as well.

The remainder of this paper is organized as follows. In Section 2, we present our problem formulation. We also describe our approach to congestion estimation in this section. In Section 3, we describe the MINOTAUR global router. We detail our methods for applying rip-up and reroute in Section 3.1, the iterative deletion approach in Section 3.2, our support for high-performance interconnect structures in Section 3.3, and the integration of algorithms in Section 3.4. Experimental results are presented in Section 4, and we conclude our paper with Section 5.

\section{Problem Formulation}

Our routing model is similar to that of [21]; we divide the 3-dimensional routing surface into a set of "tiles," with each tile consisting of a number of layers. We use "tile-layer" to indicate a single layer within a tile. The routing substrate is modeled as a graph, with tile-layers as nodes, and both vias and the "borders" between tile-layers as edges. Preferred-direction routing is obtained by including (or excluding) border edges. Figure 1 shows an example of our routing model.

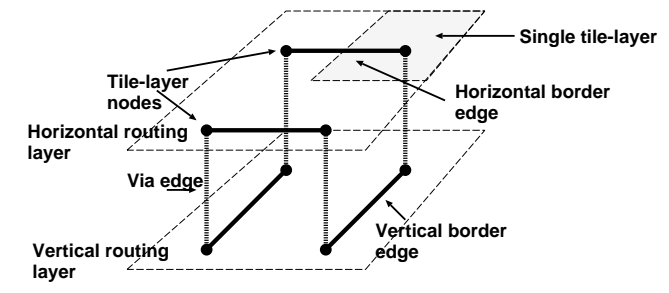

Figure 1: A two-layer four-tile routing problem.

The routing problem consists of determining the interconnect structure for a set of nets $N$. In addition to basic feasibility concerns (based on physical routing capacity constraints), there are a number of performance issues as well; many of these were detailed in [4]. In this paper, we are interested in meeting delay, signal integrity, crosstalk, and routing capacity constraints, while minimizing total wire length, maximum congestion, and routing resource costs.

We assume that a detail router will be used to complete the routings of the individual tiles, and determine solution feasibility through the maximum congestion of any tile-layer node, border edge, or via edge. We estimate solution congestion as the number of topology edges using a particular routing resource. Note that the border congestion and tilelayer congestion metrics are in general not comparable.

\section{The MINOTAUR Global Router}

Our approach is to first decompose each non-critical signal net into a set of edges. In most of our experiments, we use a simple minimum spanning tree algorithm, although we can also utilize Steiner tree or high performance interconnect algorithms. Steiner topologies had little impact on solution quality in our experiments. Nets which require highperformance interconnect structures are not decomposed into 2-pin edges, and are handled as described in Section 3.3.

For each edge of the non-critical nets, we wish to find a path through the modeled MCM substrate; we model a path as a set of tile-layer nodes, border edges, and via edges.

Our global router contains two basic algorithmic approaches: a traditional rip-up and reroute scheme, and a method based on iterative deletion. We first discuss the details of each, and then show how they are integrated.

\subsection{Rip-Up and Reroute}

Rip-up and reroute is one of the oldest approaches to the global routing problem; it generally requires long computation times and provides few guarantees as to solution quality. Despite these drawbacks, rip-up and reroute continues to be used in many routing applications. The rip-up and reroute portion of our global router is similar to that of [19], in that we iteratively change our congestion bounds to encourage convergence to a low-congestion solution.

Given the complexity of MCM and PCB designs, we believe that rip-up and reroute may be necessary for aggressive design. We implement rip-up and reroute in our router as follows. Each edge is first routed using a maze router based on Lee's classic algorithm [15]. We then iteratively rip-up and reroute each edge, continuing for a user-specified number of iterations, or until solution quality stabilizes. Details of our maze routing cost functions, and edge orderings used, are given below.

If high-performance interconnect structures are required, we select a single high-performance interconnect structure with the minimum area for the performance critical net, and do not modify it. The rip-up and reroute process operates only on the edges of non-critical nets, and attempts to find routes "around" the congestion of the critical nets. Congestion optimization of the high-performance nets by the iterative deletion method is more robust, and is detailed below.

\subsubsection{Maze Routing Cost Functions}

As is done in a number of other routers, we assign a cost to each tile-layer node, border edge, and via edge. Our objective is to assign costs to each resource such that we find a relatively short path (in terms of wiring distance), while avoiding heavily congested areas. We are therefore interested in knowing what cost functions result in the best 
performance, a question that has received relatively little attention in the literature.

In [19], the cost of routing in the most congested region is infinite, while the remaining regions have low cost. In [12], the cost of a routing path includes terms for the number of other routes that a path "touches" or "crosses," as well as two terms to capture the route length in preferred and non-preferred routing directions. In [18], steps in the preferred routing direction were assigned a cost of 1 , while non-preferred steps were assigned a cost of 30 . In [16], costs were piecewise-linear functions depending on a measure of the utilization and the capacity of each edge. Clearly, there are many possible ways to calculate the cost of using a particular resource.

In general, we expect resources which have high usage or large numbers of obstacles to have relatively high cost. We have explored three cost functions in depth, and describe them here.

We first consider a step function, similar to those of [19], [18], and [12], which provides a low cost if congestion falls below a specific threshold value. A high cost results if congestion is at or above the threshold value. Second, we considered a piece-wise linear slope function, similar to that of [16], which provides low cost when congestion is below a threshold, high cost if congestion is above cap, and a linearly interpolated cost when congestion is between the threshold and cap levels. For each iteration of rip-up and reroute, we determine the maximum and minimum congestion for any resource. We modify the step and slope functions to reflect current congestion levels by adjusting the threshold and cap parameters. We refer to this as Adaptive Congestion Estimation ( $A C E$ ), and perform experiments using $A C E$-step and $A C E$-slope cost functions. A third function, which we refer to as a "two-tier" function, is similar to the slope function when routing congestion is below a fixed physical capacity. If congestion is above physical capacity, routing costs are increased substantially. These cost functions are illustrated in Figure 2.
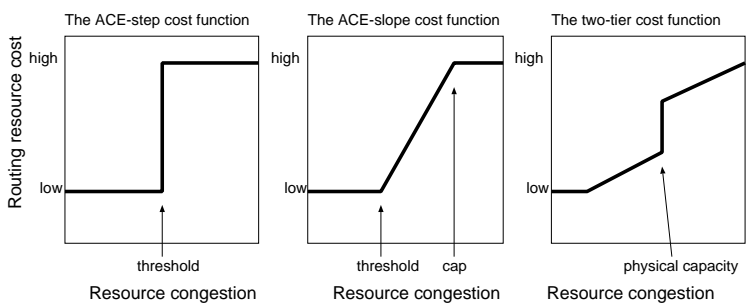

Figure 2: ACE-step, ACE-slope, and "two-tier" cost functions. These functions are used to determine resource cost during maze routing.

We find that the $A C E$-slope cost function is consistently superior to the $A C E$-step function. Detailed results are given in Section 4, Table 2. Many routing applications which utilize step-like functions ([19], [12],[18], for example) may obtain substantial improvements in results by adopting a slope-like cost function.

Our global router supports separate cost functions for each type of resource (tile-layer nodes, border edges, and via edges), and can optimize these independently.

\subsubsection{The Net Ordering Problem}

A perennial question in global routing problems has been which net do we route (or reroute) first? [9, 2]. To evaluate the importance of net ordering in our implementation, we compared five different edge orderings within the rip-up and reroute framework. Our orderings were low length first, low cost first, the reverse of these, and the default order of our design database.

Surprisingly, there was negligible difference in the results by the various orderings. While examples can be constructed in which certain orderings are clearly superior to others, we found little evidence that this occurs in our experiments. In our model, we have no hard upper limit on routing capacity, and perform rip-up and reroute until solution quality converges. Our study concludes that solution quality measured in our routing model is far more sensitive to the choice of routing cost functions than to the edge ordering.

\subsection{The Iterative Deletion Method}

Rip-up and reroute is computationally expensive. Through iterative deletion, we can obtain high quality results while using rip-up and reroute sparingly. In this section, we describe iterative deletion; Section 3.4 describes integration with rip-up and reroute. The use of iterative deletion was first proposed in [9], and was recently improved in [7].

In many iterative improvement methods, we repeatedly modify a solution, changing a small portion at each step. Rather than representing the problem with a single instance, and changing one aspect of it at each step, the iterative deletion method represents a set of possible states. Consider the example in Figure 3, which is a relatively simple planar routing problem with three nets.

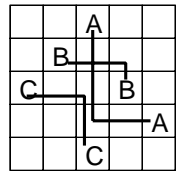

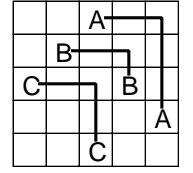

(b)

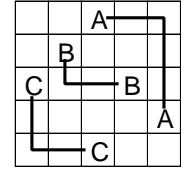

(c)

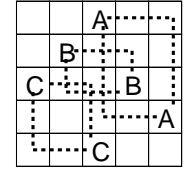

Figure 3: A simple planar routing problem (showing solutions with high congestion, high crosstalk, low crosstalk, and a set of redundant candidate routes).

Figure $3 \mathrm{a}$ has high congestion, while Figure $3 \mathrm{~b}$ has high coupling capacitance (due to parallel wire segments). Figure $3 \mathrm{c}$ has both low congestion and low coupling capacitance, and is a subset of Figure 3d. The iterative deletion process begins with a redundant set of paths (such as Figure 3d), and iteratively removes a single high-cost redundant path while attempting to minimize congestion and crosstalk among the remaining connections. Interactions between paths (such as coupling capacitance) can also be modeled within this framework.

The set of possible connections for each edge is called the path set. In Figure 3d, the path sets for each edge consist of the two single-bend routes. By having several possible routes for each edge available at the start of the iterative deletion process, we can easily determine which paths are high quality. In the example, the lower-left routing for net $C$ does not interfere with any other path, allowing the alternative to be removed without degrading solution quality. This approach has a number of significant features. First, we obtain a global estimate of congestion, and can determine not only where congestion is likely, but also where it is unavoidable. Second, crosstalk between paths can be modeled and considered during optimization. By selecting a set of routes that are compatible at a global level, we can obtain high quality solutions quickly. 


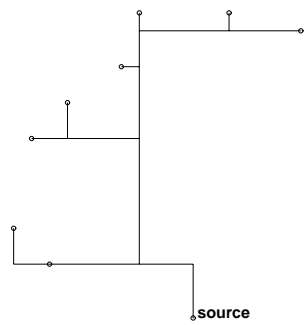

(a) Tree 1

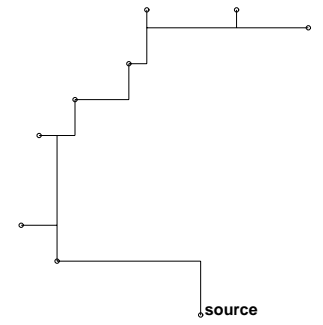

(b) Tree 2
Figure 4: An example of the topologies generated by the RATS-tree algorithm for a 9-pin net.

Iterative deletion was shown to be an effective method for selecting a set of channel segments in standard cell IC routing problems[7], and motivates our use of the method here. Our congestion estimation here is similar to the channel density estimation of [7]. We recalculate congestion levels after the removal of each redundant path.

\subsection{Performance Optimization}

Many approaches for interconnect optimization in high performance design have been proposed. [11] and [5] provide an overview of current interconnect optimization approaches. Recently, Required-Arrival-Time Steiner trees (RATS-trees) have been proposed [6] to provide a set of solutions allowing trade-off among signal delay, wave-form, and routing area. This algorithm uses a bottom-up dynamic programming approach, and can synthesize a set of high performance structures; Figure 4 shows two possible trees for a 9 -pin net.

While the our basic iterative deletion approach considers alternate routings for each topology edge, we consider alternate trees for each critical net. Rather than removing a single path between a pair of pins during deletion, we instead remove one of the candidate interconnect trees generated by the RATS-tree algorithm. The RATS-tree algorithm and our global router support variable-width routing.

\subsection{The Hybrid Approach}

We have three approaches that form a hybrid between the rip-up and reroute approach, and the iterative deletion method. The first applies iterated rip-up and reroute, and then adds the single bend paths to the path set for each edge. We then perform iterative deletion on the path sets.

Our second approach begins by generating single-bend paths for each edge, and then applies iterative deletion to these path sets. After iterative deletion, we use the maze router to find a new candidate path for each edge, and add it to the path set for the edge. Multiple iterations of this process can produce high quality results quickly.

A third approach applies iterative deletion as above, followed by rip-up and reroute for non-critical nets.

\section{Experimental Results}

Experiments were performed with the MCC multichip module benchmark circuits $m c c 1$ and mcc2, and also on the three test cases from [14], summarized in Table 1. We report maximum and average congestion levels $\left(C_{\max }\right.$ and $\left.C_{\text {avg }}\right)$ for either the tile-layer metric or the border metric (these two are

\begin{tabular}{|l|r|r|r||r|r|r|r|}
\hline \multirow{2}{*}{ Benchmark } & No. of & No. of & \multicolumn{1}{|c||}{ No. of } & \multicolumn{4}{|c|}{ Net cardinality } \\
& Chips & Nets & Pins & 2 & 3 & 4 & $5+$ \\
\hline test1 & 4 & 500 & 1000 & 500 & 0 & 0 & 0 \\
\hline test2 & 9 & 956 & 1912 & 956 & 0 & 0 & 0 \\
\hline test3 & 9 & 1254 & 2508 & 1254 & 0 & 0 & 0 \\
\hline mcc1 & 6 & 802 & 2495 & 608 & 87 & 50 & 57 \\
\hline mcc2 & 37 & 7118 & 14659 & 6699 & 415 & 4 & 0 \\
\hline
\end{tabular}

Table 1: Benchmark circuit parameters. test1, test2, and test 3 are constructed examples, containing only two-pin nets.

not in general comparable). Average congestion should correlate reasonably well with wire lengths available after completion of detail routing. A complete set of results is available in [8]. We omit experimental results on edge ordering (in which no ordering outperformed another), an exhaustive set of results comparing cost function approaches, and experiments comparing the use of spanning tree and Steiner tree topologies (which also show negligible differences).

Maze routing (which is unoptimized) dominates the run time. MINOTAUR routes mcc2 in one hour on a Sun Ultra1 , using a single layer 16 by 16 routing graph. Increasing graph resolution increases run times.

\subsection{Comparison of Cost Functions}

To compare cost function performance, we route each example as a single layer problem, using a 16 by 16 routing grid. We use a low routing cost of 1 , and tested combinations with high routing costs of $2,4,8$, and $16, A C E$ cost function threshold points at $0.2,0.4,0.6$, and 0.8 of the range between the minimum and maximum congestion level, and also a threshold point at the average congestion level. A summary of these results is shown in Table 2, in which we show the best, worst, and "typical" performance of the rip-up and reroute method for either the step or slope cost functions, and the result of a single pass of iterative deletion considering the rip-up and reroute paths and the single-bend candidate paths. In the "typical" cost function configuration, we use a high routing cost of 16 , and the cost function threshold point at the average congestion level.

While the congestion values during the rip-up and reroute process converged for all experiments, they converged to vastly different values depending on the cost function, and the high, low, and threshold values used to configure the cost function; maximum congestion levels differed by as much as a factor of 2.29. We find that the results using the slope cost function are superior to the step based cost function. When the high, low, and threshold values for tile costs are not well suited to the routing problem, a single pass of iterative deletion can improve the results considerably.

\subsection{Comparison with V4R}

In Table 3, we compare maximum tile-layer congestion values of our approach with the results of the MCM global router V4R [14]. Congestion estimation favors the V4R router, as the MINOTAUR result considers distinct tree edges, while the V4R result reported considers distinct nets. For the single layer comparisons, we merge layer assignments of the V4R routing into a single layer. Our global router obtains congestion reductions ranging from $35 \%$ to $48 \%$ for the single layer model, and reductions of $14 \%$ to $38 \%$ for the multi-layer model. 


\begin{tabular}{|c|c|c|c|c|c|c|c|c|c|c|c|c|}
\hline & \multicolumn{2}{|c|}{ Best RR } & \multicolumn{2}{|c|}{ Best RR + ID } & \multicolumn{2}{|c|}{ Worst RR } & \multicolumn{2}{|c|}{ Worst RR + ID } & \multicolumn{2}{|c|}{ Typical RR } & \multicolumn{2}{|c|}{ Typical RR + ID } \\
\hline Benchmark & $C_{\max }$ & $C_{a v g}$ & $C_{\max }$ & $C_{a v g}$ & $C_{\max }$ & $C_{a v g}$ & $C_{\max }$ & $C_{a \cup g}$ & $C_{\max }$ & $C_{a \cup g}$ & $C_{\max }$ & $C_{a v g}$ \\
\hline test1 slope & 27 & 23.7 & 27 & 23.7 & 28 & 23.7 & 28 & 23.7 & 27 & 24.3 & 28 & 24.0 \\
\hline step & 33 & 24.7 & 30 & 24.1 & 58 & 23.7 & 33 & 23.7 & 33 & 24.8 & 30 & 23.1 \\
\hline test2 slope & 49 & 46.3 & 51 & 46.1 & 51 & 46.5 & 51 & 46.2 & 50 & 47.0 & 52 & 46.5 \\
\hline step & 57 & 49.1 & 54 & 47.7 & 112 & 45.9 & 59 & 45.9 & 61 & 48.4 & 53 & 47.3 \\
\hline test3 slope & 62 & 59.0 & 64 & 59.0 & 63 & 59.9 & 65 & 59.5 & 63 & 60.3 & 65 & 59.7 \\
\hline step & 67 & 60.9 & 67 & 60.2 & 111 & 59.0 & 73 & 59.0 & 76 & 60.6 & 67 & 59.9 \\
\hline mec1 slope & 59 & 49.3 & 60 & 49.1 & 67 & 48.5 & 63 & 48.3 & 60 & 50.1 & 61 & 49.6 \\
\hline step & 83 & 51.3 & 69 & 49.6 & 123 & 48.5 & 81 & 48.5 & 89 & 51.5 & 68 & 49.9 \\
\hline mcc2 slope & 295 & 216.0 & 291 & 211.7 & $\overline{363}$ & 186.3 & $\overline{344}$ & 186.0 & $\overline{303}$ & 215.6 & 293 & 210.9 \\
\hline step & 350 & 209.1 & 312 & 203.8 & 524 & 192.1 & 376 & 188.0 & 416 & 212.3 & 356 & 205.2 \\
\hline
\end{tabular}

Table 2: Experiments on MCM benchmarks considering a variety of cost functions. We report tile-layer maximum and average congestion $C_{\max }$ and $C_{\text {avg }}$, and show the best and worst performance using a classic rip-up and reroute approach, and the results of applying a single pass of iterative deletion to the rip-up and reroute result combined with the two single-bend candidate paths. We also show results using a "typical" cost function configuration.

\begin{tabular}{|l||r|r||r|r|r|}
\hline \multicolumn{1}{|c||}{} & \multicolumn{3}{c||}{ Single Layer } & \multicolumn{3}{c|}{ Multi Layer } \\
Benchmark & Minotaur & V4R & $L$ & Minotaur & V4R \\
test1 & 27 & 52 & 4 & 16 & 26 \\
\hline test2 & 50 & 78 & 4 & 29 & 34 \\
\hline test3 & 63 & 102 & 4 & 33 & 45 \\
\hline mcc1 & 60 & 94 & 4 & 48 & 74 \\
\hline mcc2 & 303 & 496 & 6 & 196 & 228 \\
\hline
\end{tabular}

Table 3: Comparison of maximum tile-layer congestion of the MINOTAUR global routing result with that of V4R. For the single-layer comparison, the V4R layers were merged into a single layer.

\subsection{Experiments with Physical Capacity Constraints}

Table 4 shows experimental results and routings using a "two-tier" cost function and differing physical routing capacity constraints. Assuming a wire pitch of $75 \mu$, we have a physical capacity per border of 36 for mcc 1 and 125 for mcc. We also consider cases with increased wire pitch, resulting in physical capacities of 24, 20, 16, and 10 for $m c c 1$, and 100,80 , and 60 for $m c c 2$, respectively. We apply either an $A C E$-slope cost function, the "two-tier" function, or an $A C E$-slope approach followed by a post-processing step using the "two-tier" cost function. When physical constraints are relatively loose, using a two-tier cost function or $A C E$ slope followed by post-processing can further improve the averge congestion (and expected wire length), as shown in the case with a routing capacity of 125 for mcc. When an unrealistically tight capacity constrnt is given (as is the case for the capacity of 60 for mcc2) the two-tier cost function may give a solution that is inferior to the one obtained using ACE-slope.

\subsection{Performance Optimization}

To evaluate the impact of performance optimization on global solution quality, we applied the RATS-tree algorithm to a number of nets from the benchmarks $m c c 1$ and $m c c 2$. The number of nets optimized, minimum and maximum number of RATS-trees, and average number of RATS-trees per net are shown in Table 5. For these experiments, we constructed Steiner trees for each net, and evaluated the delay for each using SPICE. T\% of the pins with highest delay were selected, and we attempt to obtain an $X \%$ reduction in signal delay for these pins (no delay constraints are placed on the other nets or pins). Router performance is shown in Table

\begin{tabular}{|l|r|r|r|r|}
\hline \multicolumn{5}{|c|}{$m c c 1$} \\
& $C_{m a x}$ & $C_{a v g}$ & Mono. & Non-Mono. \\
\hline ACE-slope & 17 & 11.4 & 1597 & 97 \\
\hline \hline two-tier (35) & 27 & 11.2 & 1678 & 16 \\
\hline two-tier (24) & 21 & 11.2 & 1652 & 42 \\
\hline two-tier (20) & 17 & 11.3 & 1622 & 72 \\
\hline two-tier (16) & 16 & 11.7 & 1515 & 179 \\
\hline two-tier (10) & 22 & 11.6 & 1535 & 159 \\
\hline \hline post-process (35) & 26 & 11.2 & 1668 & 26 \\
\hline post-process (24) & 21 & 11.2 & 1645 & 49 \\
\hline post-process (20) & 17 & 11.3 & 1630 & 64 \\
\hline post-process (16) & 16 & 11.3 & 1625 & 69 \\
\hline post-process (10) & 17 & 11.4 & 1597 & 97 \\
\hline \hline \multicolumn{5}{|c|}{$m$ mcc2 $^{2}$} \\
\hline ACE-slope & 84 & 48.7 & 6874 & 667 \\
\hline \hline two-tier (125) & 103 & 43.8 & 7412 & 129 \\
\hline two-tier (100) & 84 & 44.5 & 6957 & 584 \\
\hline two-tier (80) & 81 & 48.8 & 6191 & 1350 \\
\hline two-tier (60) & 98 & 54.6 & 5406 & 2135 \\
\hline \hline post-process (125) & 103 & 43.7 & 7474 & 127 \\
\hline post-process (100) & 83 & 44.5 & 7251 & 290 \\
\hline post-process (80) & 80 & 48.8 & 6759 & 782 \\
\hline post-process (60) & 84 & 48.7 & 6874 & 667 \\
\hline
\end{tabular}

Table 4: Four-layer routing experiments on $m c c 1$ and $m c c 2$ with the border congestion metric, using physical routing capacity constraints (shown in parenthesis). We use either the $A C E$-slope cost function, a "two-tier" function, or the $A C E$-slope cost function followed by improvement with a "two-tier" function as a post-processing step. The number of monotonic and non-monotonic paths are also shown.

\begin{tabular}{|l|r||r||c||c||c|}
\hline Bench & \multicolumn{1}{|c||}{} & Total & \multicolumn{3}{c|}{ Min/max/avg. RATS-trees } \\
mark & $T$ & Nets & $X=5 \%$ & $X=10 \%$ & $X=15 \%$ \\
\hline mcc1 & $5 \%$ & 38 & $4 / 12 / 8.16$ & $4 / 12 / 8.13$ & $4 / 12 / 8.13$ \\
mcc1 & $10 \%$ & 51 & $3 / 17 / 8.71$ & $3 / 14 / 8.67$ & $3 / 14 / 8.55$ \\
mcc2 & $5 \%$ & 614 & $1 / 6 / 1.02$ & $1 / 4 / 1.02$ & $1 / 4 / 1.02$ \\
mcc2 & $10 \%$ & 1278 & $1 / 6 / 1.01$ & $1 / 4 / 1.01$ & $1 / 4 / 1.01$ \\
\hline
\end{tabular}

Table 5: Number of nets with performance constraints, and the minimum, maximum, and average number of RATStrees for each net. 


\begin{tabular}{|c|c|c|c|c|c|c|c|c|c|c|c|c|c|}
\hline \multirow{3}{*}{ Benchmark } & \multirow{3}{*}{$T$} & \multicolumn{6}{|c|}{ Rip-up and reroute } & \multicolumn{6}{|c|}{ Iterative deletion with RR postprocessing } \\
\hline & & $X=$ & $5 \%$ & $X=$ & $0 \%$ & $X=$ & $15 \%$ & $X=$ & $5 \%$ & $X=$ & $0 \%$ & $X=$ & $15 \%$ \\
\hline & & $C_{\max }$ & $C_{a v g}$ & $C_{\text {max }}$ & $C_{a \cup g}$ & $C_{\max }$ & $C_{a v g}$ & $C_{\max }$ & $C_{a v g}$ & $C_{\max }$ & $C_{a \cup g}$ & $C_{\max }$ & $C_{a v g}$ \\
\hline $\mathrm{mcc1}$ & $5 \%$ & 34 & 22.6 & 34 & 22.6 & 34 & 22.6 & 32 & 22.6 & 33 & 22.7 & 33 & 22.7 \\
\hline $\operatorname{mcc1}$ & $10 \%$ & 41 & 22.3 & 41 & 22.3 & 41 & 22.3 & $\overline{33}$ & 22.8 & 33 & 22.8 & 33 & 22.8 \\
\hline $\mathrm{mcc} 2$ & $5 \%$ & 184 & 93.6 & 184 & 93.6 & 184 & 93.6 & 184 & 93.6 & 184 & 93.6 & 185 & 93.6 \\
\hline $\mathrm{mcc} 2$ & $10 \%$ & 223 & 93.3 & 223 & 93.3 & 223 & 93.3 & 222 & 93.3 & 222 & 93.3 & 222 & 93.3 \\
\hline
\end{tabular}

Table 6: Performance optimization comparison of rip-up and reroute (using a single topology for critical nets) with iterative deletion followed by rip-up and reroute. In these experiments, we select $T \%$ of the high-delay pins, and attempt to obtain at least an $X \%$ delay reduction for each. In experiments with no performance optimization, rip-up and reroute obtains maximum and average congestion levels of 33 and 23.0 for mcc1, while congestion levels are 168 and 93.4 for mcc2. These results assume the single-layer routing model.

6.

We consider two routing approaches for this problem. The first approach selects a single minimum-area candidate RATS-tree for each constrained net, and then applies rip-up and reroute the the remaining nets. The second approach is a hybrid, and applies iterative deletion to select a single RATS-tree for each constrained net, and then applies ripup and reroute. When a number of candidate topologies are available, the hybrid approach obtains a $19.5 \%$ reduction in maximum congestion. In this set of experiments, the minimum-area RATS-trees were quite similar under the different performance constraints, resulting in similar performance for the rip-up and reroute approach.

\section{Conclusions}

We have presented an MCM/PCB global router which integrates the classic rip-up and reroute approach, with the modern iterative deletion method.

We draw a number of conclusions from our experiments. We find that slope-based cost functions are superior to stepbased cost functions, rip-up and reroute performance is heavily dependent on cost function parameters, and net ordering has little impact for our routing model. Iterative deletion is effective for congestion minimization when we have signal delay and integrity performance constraints, or when rip-up and reroute cost function parameters are not well chosen.

We are currently integrating our MCM global router with a performance driven variable width multi-layer detail router, and will report results of these experiments when they are complete. We are also considering the use of a small set of candidate routes such as those suggested in [2], allowing us to eliminate maze routing entirely. Our methods may also extend to handle general area routing for performance driven multi-layer VLSI IC design problems.

\section{Acknowledgements}

This work is partially supported by DARPA/ETO under Contract DAAL01-96-K-3600 managed by the US Army Research Laboratory. The authors would like to thank Mr. Cheng-Kok Koh for his assistance and for supplying performance constraints and the RATS-tree algorithm.

\section{References}

[1] H. B. Bakoglu. Circuits, Interconnections, and Packaging for VLSI. Addison-Wesley, 1990.

[2] J. D. Carothers, D. Li, and T. Hameenanttila. MCG: A correctby-design multichip module router with crosstalk avoidance. In Int. Workshop on Rapid System Prototyping, pages 183-188, 1996
[3] Y.-J. Cha, C. S. Rim, and K. Nakajima. A simple and effective greedy multilayer router for MCMs. In Proc. Int. Symp. on Physical Design, pages 67-72, 1997.

[4] J. D. Cho, K.F. Liao, S. Raje, and M. Sarrafzadeh. $M^{2} R$ : Multilayer routing algorithm for high-performance MCMs. IEEE Trans. on Circuits and Systems, 41(4):253-265, April 1994.

[5] J. Cong, L. He, C.-K. Koh, and P. H. Madden. Performance optimization of VLSI interconnect layout. Integration, the VLSI Journal, 21:1-94, 1996

[6] J. Cong and C.-K. Koh. Interconnect layout optimization under higher-order RLC model. In Proc. Int. Conf. on Computer Aided Design, 1997.

[7] J. Cong and P. H. Madden. Performance driven routing for standard cell design. In Proc. Int. Symp. on Physical Design, pages $73-80,1997$

[8] J Cong and P. H. Madden Performance driven multi-layer general area routing for $\mathrm{pcb} / \mathrm{mcm}$ designs,. Technical Report 980013, UCLA CS Dept, March 1998

[9] J. Cong and B. Preas. A new algorithm for standard cell global routing. In Proc. Int. Conf. on Computer Aided Design, pages 176-179, November 1988.

[10] A. Hanafusa, Y. Yamashita, and M. Yasuda. Three-dimensional routing for multilayer ceramic printed circuit boards. In Proc. Int. Conf. on Computer Aided Design, pages 386-389, 1990.

[11] A. B. Kahng and G. Robins. On Optimal Interconnections for VLSI. Kluwer Academic Publishers, 1994

[12] K. Kawamura, T. Shindo, T. Shibuya, H. Miwatari, and Y. Ohki Touch and cross router. In Proc. Int. Conf. on Computer Aided Design, pages 56-59, 1990.

[13] K.-Y. Khoo and J. Cong. A fast multilayer general area router for MCM designs. IEEE Trans. on Circuits and Systems, pages $841-851,1992$.

[14] K.-Y. Khoo and J. Cong. An efficient multilayer MCM router based on four-via routing. IEEE Trans. on Computer-Aided Design of Integrated Circuits and Systems, pages 1277-1290, 1994

[15] C. Y. Lee. An algorithm for path connection and its applications IRE Trans. on Electronic Computers, EC-10(3):346-365, 1961

[16] R. Linsker. An iterative-improvement penalty-function-driven wire routing system. IBM J. Research and Development, 28(5):613-624, September 1984

[17] S. Mehrotra, P. Franzon, and M. Steer. Performance driven global routing and wiring rule generation for high speed PCBs and MCMs. In Proc. Design Automation Conf, pages 381-387, 1995

[18] H. Shin and A. Sangiovanni-Vincentelli. A detailed router based on incremental routing modifications: Mighty. IEEE Trans. on Computer-Aided Design of Integrated Circuits and Systems, CAD-6(6):942-955, November 1987

[19] E. Shragowitz and S. Keel. A global router based on a multicommodity flow model. Integration, the VLSI Journal, 5:3-16, 1987

[20] D. Wang and E. S. Kuh. A new timing-driven multilayer MCM/IC routing algorithm. In Proc. IEEE Multi-Chip Module Conf., pages 89-94, 1997.

[21] Q. Yu, S. Badida, and N. Sherwani. Algorithmic aspects of three dimensional MCM routing. In Proc. Design Automation Conf, pages $397-401,1994$ 